\title{
Drug release from bone implants in-vitro: An experimental setup
}

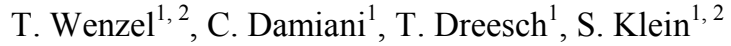 \\ ${ }^{1}$ Medical Sensors and Devices Laboratory, Luebeck University of Applied Sciences, Luebeck, Germany \\ ${ }^{2}$ Centre of Excellence for Technology and Engineering in Medicine (TANDEM), Luebeck, Germany \\ e-Mail: tobias.wenzel@fh-luebeck.de
}

Keywords: drug delivery; in-vitro model; drug eluting implants, orthopaedic implants

\begin{abstract}
Orthopaedic implants are widely used as drug carriers for local drug delivery to minimize infection risks for the patients after surgery. The drug release kinetics of those implants should ideally be assessed in-vivo through animal or human trials, which are expensive and time-consuming. In-vitro testing is a more affordable and simpler alternative. Conventional in-vitro methods are mostly too simplistic (e.g. paddle apparatus); they disregard the complex in-vivo situation and do not allow a valid in-vitro/in-vivo correlation. In this work, a new in-vitro method is proposed, in which drug diffusion and transport at the bone-implant interface can be taken into account by using a hydrogel membrane and a flow cell. The amount of released drug can be monitored continuously using a closed loop configuration. First tests using fluorescinsodium as model drug were carried out. The total amount of released drug after seven days was $16.6 \mu \mathrm{g}$. The release rate remained constant at $0.1 \mu \mathrm{g} / \mathrm{h}$ over the first four days and started decreasing afterwards, probably due to the falling concentration in the main drug reservoir.
\end{abstract}

\section{$1 \quad$ Introduction}

Coating of orthopaedic implants with bioactive substances represents a promising approach for targeted drug delivery in traumatology and orthopaedic surgery [1]. Antibiotics, for example, can be used to inhibit biofilm formation and to prevent implant induced infections [2]. Osseointegration of orthopaedic devices can be improved by coating with proteins [3]. The drug release kinetics of such implants has to be extensively assessed in-vitro and in-vivo. In-vivo testing, however, implies animal trials and clinical studies, which are ethically questionable as well as timeconsuming and expensive. The most common in-vitro method to investigate the drug release from medical devices is the so called "paddle apparatus" $[4,5]$. An implant model is immersed in a defined volume of a buffer solution (e.g. Phosphate buffered saline, PBS). Stirring of the solution facilitates the homogeneous distribution of the released drug in the buffer. Samples are taken at defined time intervals and replaced by fresh buffer. Drug concentrations in the samples are then determined using standard assays (e.g. UV/VIS spectrometry).

Limitations of this and other in-vitro methods are founded in the simplification of the much more complex in-vivo situation, resulting in a poor prediction of the drug release kinetics. Implantation of an orthopaedic device into bone damages blood vessels, nerves, tendons and bone tissue. Blood is the first tissue that is in contact with the implant after surgery. Blood proteins are then adsorbed on the material's surface and the implant becomes encapsulated by clotted blood. The blood clot changes within the first couple of days as part of the normal wound healing processes and the ossification processes begin (see figure 1) [6]. The resulting conditions at the implant's interface represent a key factor regarding the release kinetics of a drug eluting implant [7].

This work describes a novel in-vitro method that can be used for continuous monitoring of the release kinetics of drug eluting orthopaedic implants. The tissue-implant boundary is modelled by using a hydrogel membrane as diffusion barrier. Drug transport through blood vessels is simulated using a flow channel.

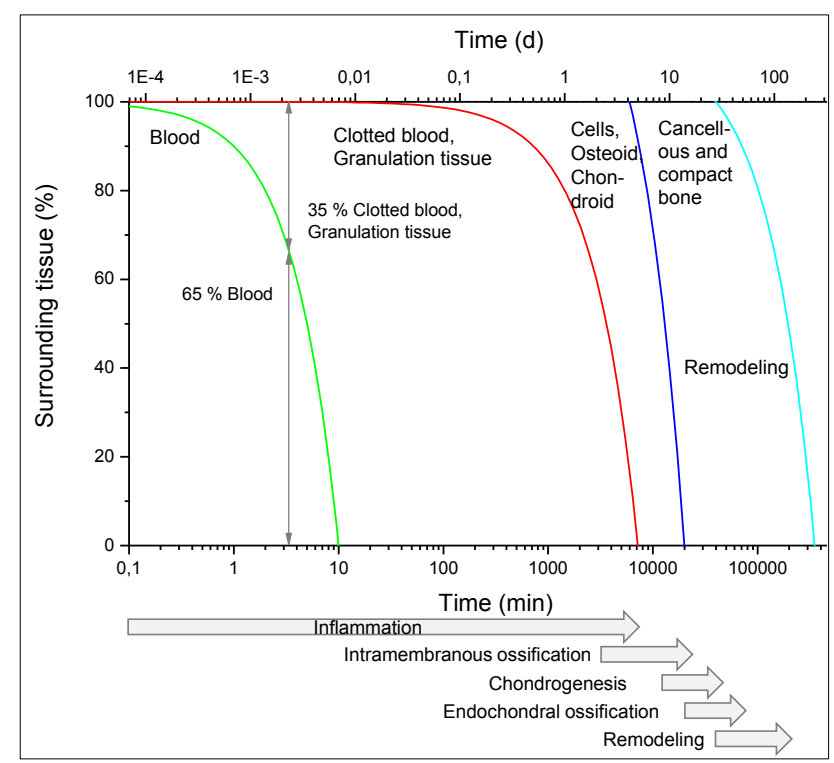

Figure 1 Wound healing in bones 


\section{Methods}

\subsection{Materials}

Hydrogels (2-hydroxyethylmethacrylate, water amount $66 \%$ ) were obtained from Wöhlk-Contact-Linsen $\mathrm{GmbH}$ (Schoenkirchen/Kiel, Germany) in the form of cylindrical rods. Slices with different thicknesses were cut from the rods using a turning machine. Swelling of hydrogels was achieved by overnight immersion in phosphate buffered saline (PBS, pH 7.4). Fluorescein sodium salt (SigmaAldrich GmbH, Taufkirchen, Germany) was used as the model drug.

\section{$2.2 \quad$ Experimental setup}

The model for in-vitro drug release (see fig. 2) comprises of a compartment containing the test sample (e.g. coated implant model or test drug solution) which is separated from a flow channel by the hydrogel membrane. As shown in fig. 3, the model is connected to a peristaltic pump and a fluorescence flow-through cuvette in a closed-loop configuration.

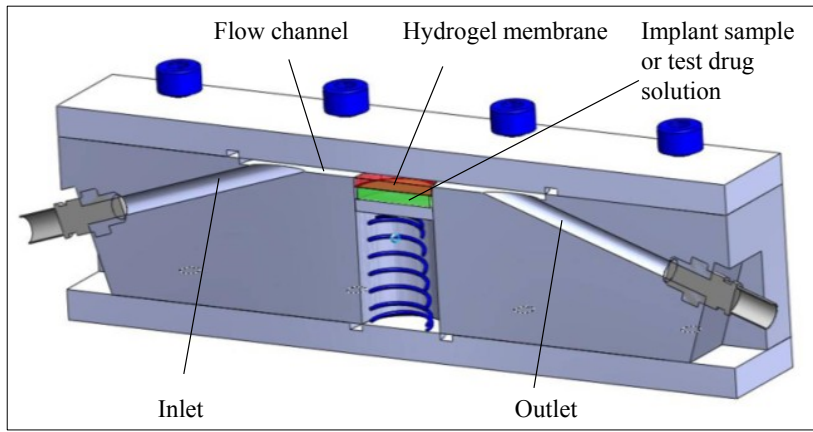

Figure 2 Flow cell

The test drug diffuses through the hydrogel membrane into the flow channel and its concentration is monitored continuously by fluorescence spectroscopy (Spectrometer Avantes 2048-L, LED light source AvaLight 475, Avantes, Netherlands). The spectrometer and the light source are both kept at a constant temperature $\left(28^{\circ} \mathrm{C} \pm 0.8^{\circ} \mathrm{C}\right)$. The fluorescent values are calculated by averaging ten single spectra and then integrating the resulting curve from wavelengths of $480 \mathrm{~nm}$ to $610 \mathrm{~nm}$.

Calibration of the experimental setup prior to the measurements was done using different concentrations of the test drug (fluorescein sodium) in PBS. Fluorescein is known to decrease its fluorescence activity over time if exposed to light [8]. Tests confirmed a continuous decrease of the fluorescence signal for solutions stored under normal room conditions (see fig. 4). All fluidic components were thus covered with aluminum foil to ensure stable long-term measurements.

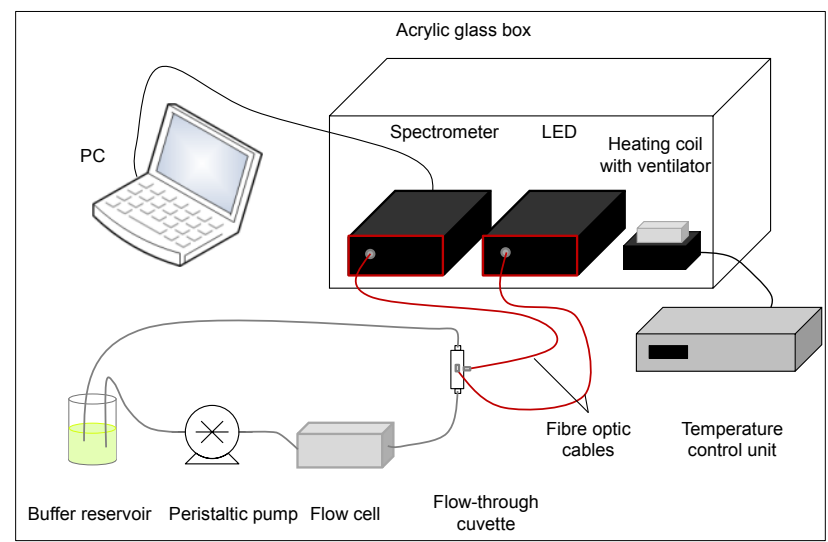

Figure 3 Experimental setup

As the initial iteration, the flow velocity in the in-vitro model was set to $0.5 \mathrm{~mm} / \mathrm{s}$ (equals $0.3 \mathrm{ml} / \mathrm{min}$ ) to approximate blood velocity in capillaries.

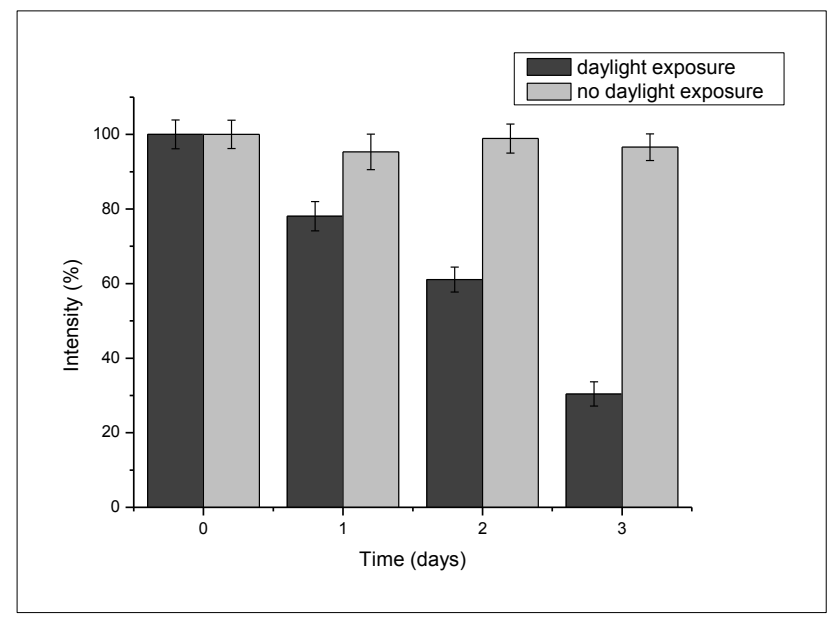

Figure 4 Long-term intensity stability of a $1 \mu \mathrm{M}$ fluorescein solution. Shown are mean values \pm standard deviations.

\section{Results}

\subsection{Calibration of the experimental setup}

Figure 5 displays the calibration curve of the experimental setup for fluorescein sodium. The lowest and highest quantification limits were $250 \mathrm{nM}$ and $50 \mu \mathrm{M}$, respectively. The precision of the experimental procedure was found to be between $0.04 \%$ and $4 \%$ (concentration dependent). The accuracy lies between $6 \%$ and $18 \%$ (concentration dependent). 


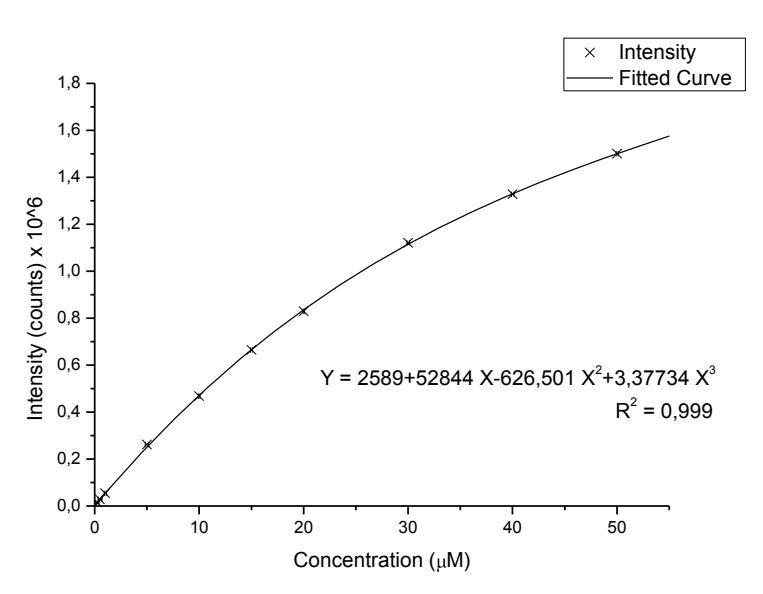

Figure 5 Calibration of the setup

\subsection{In-vitro-release studies}

A freshly prepared $40 \mu \mathrm{M}$ fluorescein sodium aqueous solution was used as the test drug. The measured concentrations as well as the corresponding amounts of released drug vs. time are shown in figure 6.

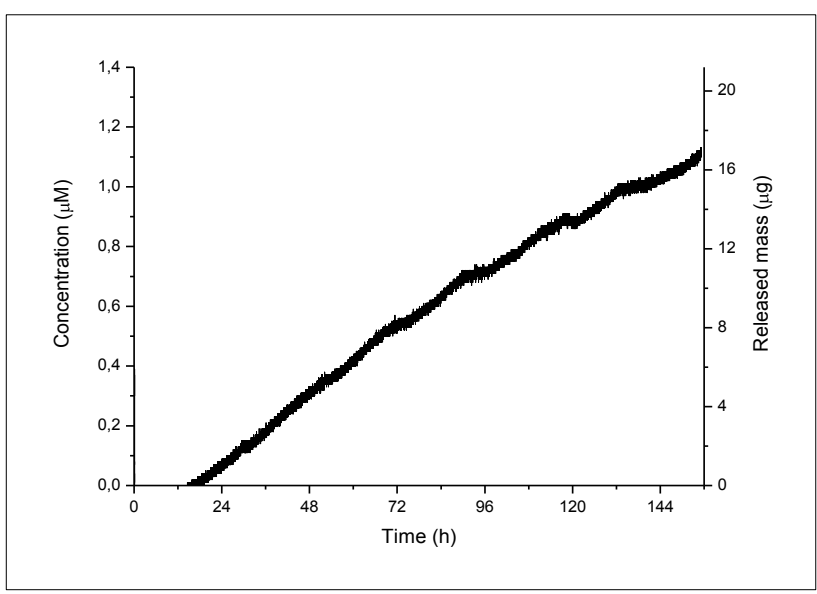

Figure 6 Concentration vs. time

The first detectable dye concentrations were measured after 12 hours. After the first week the test drug concentration in the main flow circuit was $1.1 \mu \mathrm{M}$, which equals an amount of released drug of $16.6 \mu \mathrm{g}(40 \%$ of the available amount in the reservoir).

The test drug concentration in the main flow circuit increased almost linearly within the first four days at about $0.1 \mu \mathrm{g} / \mathrm{h}$, but seemed to taper off afterwards. This was due to the decrease of the concentration gradient across the hydrogel barrier due to the drug leaving the reservoir.

Small oscillations $( \pm 10 \%)$ due to the night and day temperature fluctuations can be observed on the curve.

\section{Conclusion}

The proposed method can be used for continuous monitoring of the drug release process. Future measurements will be carried out with coated implant models. Parameters like membrane thickness or flow rate should then be tuned to match in-vivo data reported in the literature.

\section{Acknowledgements}

We acknowledge the support of the European Union and the state of Schleswig-Holstein (Programme for the Future-Economy: 122-09-024). This work was also supported by Stryker Trauma GmbH, Schoenkirchen/Kiel, Germany.

\section{References}

[1] Arruebo, M. et al.: Drug delivery from internally implanted biomedical devices used in traumatology and in orthopedic surgery. Expert Opin. Drug Deliv., 7(5), 2010

[2] Zilberman, M. and Elsner, J.: Antibiotic eluting medical devices for several applications. Journal of controlled release, Vol. 130, 2008

[3] Shi, X. et al.: A protein/antibiotic releasing poly(lactic-co-glycolic acid)/lecithin scaffold for bone repair applications. International Journal of Pharmaceutics, Vol. 373, 2009

[4] Ramchandani, M. and Robinson, D. In vitro and in vivo release of ciprofloxacin from PLGA 50:50 implants. Journal of Controlled Release, Vol. 54, 1998

[5] Sanchez, E. et al. In vitro - in vivo study of biodegradable and osteointegrable gentamicin bone implants. European Journal of Pharmaceutics and Biopharmaceutics, Vol. 52, 2001

[6] Wintermantel, E. and Ha, S.-W. Medizintechnik - Life Science Engineering, Springer, 2009

[7] Lee, C.G. et al. Simulation of Gentamicin Delivery for the Local Treatment of Osteomyelitis, Biotechnology and Bioengineering, Vol. 91, 2005

[8] Smith, SA. and Pretorius, WA: The conservative behaviour of fluorescein. Water $S A$, Vol. 28, October 2002 